J. Hano

Nagoya Math. J.

Vol. 61 (1976), 197-202

\title{
A GEOMETRICAL CHARACTERIZATION OF A CLASS OF HOLOMORPHIC VECTOR BUNDLES OVER A COMPLEX TORUS
}

\author{
JUN-ICHI HANO*)
}

This note is to be a supplement of the preceeding paper in the journal by Matsushima, settling a question raised by him. In his paper he associates a holomorphic vector bundle over a complex torus to a holomorphic representation of what he calls Heisenberg group. We shall show that a simple holomorphic vector bundle is determined in this manner if and only if the associated projective bundle admits an integrable holomorphic connection. A theorem by Morikawa ([3], Theorem 1) is the motivation of this problem and is somewhat strengthened by our result.

Let $V$ be a complex vector space of dimension $n$ and let $L$ be a lattice in $V$. The quotient group $V / L=E$ is a complex torus. It is known ([2], §3) that a holomorphic vector bundle $F$ of $\operatorname{rank} m$ over $E$ is determined by a $G L(m, C)$-valued theta factor $J$, namely by a holomorphic map

$$
J: L \times V \rightarrow G L(m, C)
$$

satisfying the following equality:

$$
J(\alpha+\beta, u)=J(\alpha, \beta+u) J(\beta, u) \quad \text { for } \alpha, \beta \in L \text { and } u \in V .
$$

We denote by $F_{J}$ the holomorphic vector bundle over $E$ determined by a theta factor $J$.

A résumé of Matsushima's construction of holomorphic vector bundles over $E$ is in order. Let $H$ be a hermitian form on $V \times V$. Let $G_{H}$ be a nilpotent Lie group whose underlying manifold is $V \times C^{*}$ and whose multiplication is defined by

Received November 25, 1975.

*) Partially supported by N.S.F. Grant GP-34710. 


$$
(u, c) \cdot(v, d)=\left(u+v, e\left[\frac{1}{2 i} H(u, v)\right] c d\right) \quad \text { for }(u, c),(v, d) \in V \times C^{*}
$$

where $e[x]=\exp 2 \pi i x$.

We denote by $G_{H}(L)$ the subgroup $L \times C^{*}$ in $G_{H}$, which is a complex Lie group. The right action of the complex Lie group $G_{H}(L)$ on the complex manifold $V \times C^{*}$ is holomorphic. Thus $V \times C^{*}$ is a holomorphic principal bundle over $E$ with structure group $L \times C^{*}=G_{H}(L)$.

If a holomorphic representation $\rho: G_{H}(L) \rightarrow G L(m, C)$ is given, a holomorphic vector bundle $F_{H}, \rho$ is determined as the quotient space $V \times C^{*} \times C^{m} /\left\{G_{H}(L), \rho\right\}$. Lemma 3.1 in [2] shows that a theta factor $J_{\rho}$ associated to the holomorphic vector bundle $F_{H}, \rho$ is given by

$$
J_{\rho}(\alpha, u)=\rho\left(-\alpha, e\left[\frac{1}{2 i}(H(u, \alpha)+H(\alpha, \alpha))\right]\right) \quad \text { for } \alpha \in L, u \in V .
$$

THEOREM. Suppose that the associated projective bundle of a holomorphic vector bundle $F$ over a complex torus $E$ admits an integrable holomorphic connection, or equivalently admits a system of transition functions which are constant. Then, we can choose a hermitian form $H_{1}$ on $V \times V$ whose imaginary part assumes rational values on $L \times L$, and a holomorphic representation $\rho$ of $G_{H_{1}}(L)$ so that $F$ is isomorphic to $F_{H_{1}}, \rho$.

Proof. (a) It is well known (Atiyah [1], Proposition 14) that the associated projective bundle $P(F)$ of a holomorphic vector bundle $F$ admits an integrable holomorphic connection if and only if $P(F)$ arises from a homomorphism $h$ of the fundamental group $L$ of torus $E$ into $P G L(m, C)$. A necessary and sufficient condition for the projective bundle $P(F)$ to have an integrable holomorphic connection is that one can choose a theta factor $J$ of $F$ such that

$$
J(\alpha, u)=J(\alpha, 0) \mu(\alpha, u)
$$

with scalar function $\mu(\alpha, u)$ for each $\alpha \in L$.

Indeed, this condition is sufficient. Suppose that $P(E)$ admits an integrable holomorphic connection. Then $P(E)$ arises from a homomorphism $h: L \rightarrow P G L(m, C)$. Let us denote by $\tilde{J}(\alpha, u)$ the image of a theta factor $J(\alpha, u)$ under the natural homomorphism of $G L(m, C)$ onto $P G L(m, C)$. Since the $P G L(m, C)$-valued factor $\tilde{J}$ and the homomorphism $h$ define the same bundle $P(F)$, 


$$
h(\alpha)=\tilde{\varphi}(u+\alpha) \tilde{J}(\alpha, u) \tilde{\varphi}(u)^{-1}
$$

with a $P G L(m, C)$-valued holomorphic function $\tilde{\varphi}$ on $V$. Since $V$ is simply connected, we can lift $\tilde{\varphi}$ to a holomorphic map $\varphi: V \rightarrow S L(m, C)$ so that $\varphi(u)$ is lying above $\tilde{\varphi}(u)$. Then, $J^{\prime}(\alpha, u)=\varphi(u+\alpha) J(\alpha, u) \varphi(u)^{-1}$ is a theta factor with required property.

(b) Let us assume that a holomorphic vector bundle $F$ satisfies the condition in the theorem and that a theta factor $J$ of $F$ is chosen so that the condition (3) is satisfied. From the condition (1) on $J$, it follows that the scalar function $\mu$ determined by (3) satisfies the following equalities :

(i ) $\mu(\alpha, \beta) \mu(\alpha+\beta, u)=\mu(\alpha, \beta+u) \mu(\beta, u), \quad$ for $\alpha, \beta \in L, u \in V$;

(ii) $\mu(\alpha, 0)=\mu(0, u)=1$, for $\alpha \in L, u \in V$;

(iii) $\mu(\alpha,-\alpha)=\mu(-\alpha, \alpha), \alpha \in L$.

We define a multiplication $\times$ on $L \times C^{*}$ in terms of $\mu$ and make $L \times C^{*}$ a complex Lie group $G_{\mu}(L)$ :

$$
(\alpha, c) \times(\beta, d)=(\alpha+\beta, \mu(\beta, \alpha) c d) \quad \text { for }(\alpha, c),(\beta, d) \in L \times C^{*} .
$$

The associative law is verified by (i). The identity is $(0,1)$, because of (ii) and the inverse of $(\alpha, c)$ is $\left(-\alpha, \mu(-\alpha, \alpha)^{-1} c\right)$.

Define a map

$$
f: G_{\mu}(L) \rightarrow G L(m, C)
$$

by $f(\alpha, c)=J(\alpha, 0)^{-1} c$. Then, $f$ is a holomorphic representation. In fact,

$$
f((\alpha, c) \times(\beta, d))=J(\alpha+\beta, 0)^{-1} \mu(\beta, \alpha) c d .
$$

Since $J(\alpha+\beta, 0)=J(\beta, \alpha) J(\alpha, 0)=J(\beta, 0) J(\alpha, 0) \mu(\beta, \alpha)$ by (1) and (3),

$$
\begin{aligned}
f((\alpha, c) \times(\beta, d)) & =J(\alpha, 0)^{-1} J(\beta, 0)^{-1} c d \\
& =f(\alpha, c) f(\beta, d) .
\end{aligned}
$$

(c) The map $L \times V \rightarrow C^{*}$ given by $(\alpha, u) \rightarrow \operatorname{det} J(\alpha, u)$ is a $C^{*}$-valued theta factor corresponding to the line bundle $\operatorname{det} F$, which is equivalent to a normalized theta factor ([4], p. 111). We choose a $C^{*}$-valued holomorphic function $\varphi$ on $V$, a hermitian form $H$ on $V \times V$ whose imaginary part assumes integral values on $L \times L$ and a semi-character $\chi: L \rightarrow C^{*}$ such that

$$
\operatorname{det} J(\alpha, u)=\varphi(u+\alpha) \chi(\alpha) e\left[\frac{1}{2 i} H(u, \alpha)+\frac{1}{4 i} H(\alpha, \alpha)\right] \varphi(u)^{-1}
$$


On the other hand from (3),

$$
\operatorname{det} J(\alpha, u)=\operatorname{det} J(\alpha, 0) \mu^{m}(\alpha, u) \text {. }
$$

Thus,

$$
\operatorname{det} J(\alpha, 0)=\varphi(\alpha) \varphi(0)^{-1} \chi(\alpha) e\left[\frac{1}{4 i} H(\alpha, \alpha)\right]
$$

and

$$
\mu^{m}(\alpha, u)=\varphi(u+\alpha) \varphi(\alpha)^{-1} e\left[\frac{1}{2 i} H(u, \alpha)\right] \varphi(u)^{-1} \varphi(0)
$$

Since $\varphi$ is a nowhere vanishing holomorphic function on a simply connected space $V$, there is a nowhere vanishing holomorphic function $\psi$ on $V$ such that $\psi^{m}=\varphi$. For each $\alpha$, an $m^{\text {th }}$ root of unity $\varepsilon_{\alpha}$ is determined by

$$
\mu(\alpha, u)=\varepsilon_{\alpha} \psi(u+\alpha) \psi(\alpha)^{-1} e\left[\frac{1}{2 m i} H(u, \alpha)\right] \psi(u)^{-1} \psi(0) .
$$

Putting $u=0$, we see that $1=\mu(\alpha, 0)=\varepsilon_{\alpha}$. Thus,

$$
\mu(\alpha, u)=\frac{\psi(u+\alpha)}{\psi(\alpha)} e\left[\frac{1}{2 m i} H(u, \alpha)\right] \frac{\psi(0)}{\psi(u)} .
$$

(d) The above relation enables us to establish an isomorphism of $G_{H / m}(L)$ and $G_{\mu}(L)$. Put

$$
\lambda(\alpha)=\psi(\alpha) / \psi(0)
$$

Then from (5),

$$
\mu(\alpha, \beta)=\frac{\lambda(\alpha+\beta)}{\lambda(\alpha) \lambda(\beta)} e\left[\frac{1}{2 m i} H(\beta, \alpha)\right]
$$

Making use of the $\lambda(\alpha)$ 's, we define a map

$$
g: G_{H / m}(L) \rightarrow G_{\mu}(L)
$$

by

$$
g(\alpha, c)=(\alpha, \lambda(\alpha) c), \quad(\alpha, c) \in L \times C^{*} .
$$

We claim that $g$ is an isomorphism. Obviously, $g$ is $1: 1$ and onto. 


$$
\begin{aligned}
g((\alpha, c) \cdot(\beta, d)) & =g\left(\alpha+\beta, e\left[\frac{1}{2 m i} H(\alpha, \beta)\right] c d\right) \\
& =\left(\alpha+\beta, \lambda(\alpha+\beta) e\left[\frac{1}{2 m i} H(\alpha, \beta)\right] c d\right) \\
& =(\alpha+\beta, \lambda(\alpha) \lambda(\beta) \mu(\beta, \alpha) c d) \\
& =g(\alpha, c) \times g(\beta, d),
\end{aligned}
$$

on account of (6). Thus $g$ is an isomorphism.

(e) From (4) and (7), $\rho=f \circ g$ is a holomorphic representation of $G_{H / m}(L)$ into $G L(m, C)$ given by

$$
\rho(\alpha, c)=J(\alpha, 0)^{-1} \lambda(\alpha) c .
$$

The theta factor $J^{\prime}$ associated to the representation $\rho$ in the formula (2) is

$$
\begin{aligned}
J^{\prime}(\alpha, u) & =\rho\left(-\alpha, e\left[\frac{1}{2 m i}(H(u, \alpha)+H(\alpha, \alpha))\right]\right) \\
& =J(-\alpha, 0)^{-1} \lambda(-\alpha) e\left[\frac{1}{2 m i}(H(u, \alpha)+H(\alpha, \alpha))\right] .
\end{aligned}
$$

Making use of the equalities

$$
\begin{aligned}
& J(-\alpha, 0)^{-1}=J(\alpha, 0) \mu(0,-\alpha) \\
& \mu(\alpha,-\alpha)=\lambda(\alpha)^{-1} \lambda(-\alpha)^{-1} e\left[-\frac{1}{2 m i} H(\alpha, \alpha)\right]
\end{aligned}
$$

and of the equality (5), we see that

$$
J^{\prime}(\alpha, u)=\psi(u+\alpha)^{-1} J(\alpha, u) \psi(u) .
$$

Thus, we have seen that the theta factors $J$ and $J^{\prime}$ are equivalent and hence $F \cong F_{H / m}, \rho$, finishing the proof.

Remark. In order to prove the converse of the theorem, we assume that a holomorphic vector bundle $F_{\rho}$ over $E$ associated to a holomorphic representation $\rho$ of $G_{H}(L)$ is simple. Then, the image of the central subgroup $\{0\} \times C^{*}$ is of scalar matrices and hence $\rho(0, c)=c^{k}$ for some integer $k$. The projective representation $\tilde{\rho}$ reduces to a projective representation of $L$. By Atiyah's proposition, $P\left(F_{\rho}\right)$, which arises from $\tilde{\rho}$ of $L$, admits an integrable holomorphic connection. 


\section{BIBLIOGRAPHY}

[1] Atiyah, M. F., Complex analytic connections in fibre bundles, Trans. AMS. 85 (1957), 181-207.

[2] Matsushima, Y., Heisenberg groups and holomorphic vector bundles over a complex torus, Nagoya Math. J. 61 (1976).

[ 3 ] Morikawa, H., A note on holomorphic vector bundles over complex tori, Nagoya Math. J. 41 (1971), 101-106.

[ 4 ] Weil, A., Introduction à l'etude des variétés kahleriennes, Paris, Hermann (1958).

Washington University 